\title{
TITLE:
}

\section{A corpuscular picture of electrons in chemical bond.}

$\operatorname{AUTHOR}(S):$

Ando, Koji

\section{CITATION:}

Ando, Koji. A corpuscular picture of electrons in chemical bond.. The Journal of chemical physics 2016, 144(12): 124109.

\section{ISSUE DATE:}

2016-03-25

URL:

http://hdl.handle.net/2433/210098

\section{RIGHT:}

Copyright 2016 AIP Publishing. This article may be downloaded for personal use only. Any other use requires prior permission of the author and AIP Publishing. The following article may be found at http://scitation.aip.org/content/aip/journal/jcp/144/12/10.1063/1.4944 827. 


\section{AIP | The Journal of}

\section{A corpuscular picture of electrons in chemical bond}

Koji Ando

Citation: The Journal of Chemical Physics 144, 124109 (2016); doi: 10.1063/1.4944827

View online: http://dx.doi.org/10.1063/1.4944827

View Table of Contents: http://scitation.aip.org/content/aip/journal/jcp/144/12?ver=pdfcov

Published by the AIP Publishing

\section{Articles you may be interested in}

Valence-bond non-equilibrium solvation model for a twisting monomethine cyanine

J. Chem. Phys. 142, 084502 (2015); 10.1063/1.4907758

Generalized valence bond wave functions in quantum Monte Carlo

J. Chem. Phys. 132, 164110 (2010); 10.1063/1.3377091

Tractable spin-pure methods for bond breaking: Local many-electron spin-vector sets and an approximate valence bond model

J. Chem. Phys. 130, 084103 (2009); 10.1063/1.3069296

Gold as hydrogen: Structural and electronic properties and chemical bonding in Si 3 Au $3+/ 0 /-$ and comparisons to $\mathrm{Si} 3 \mathrm{H} 3+/ 0 /-$

J. Chem. Phys. 125, 133204 (2006); 10.1063/1.2216707

Ab initio calculation of the $\mathrm{NH}(\Sigma-3)-\mathrm{NH}(\Sigma-3)$ interaction potentials in the quintet, triplet, and singlet states

J. Chem. Phys. 123, 184302 (2005); 10.1063/1.2079867

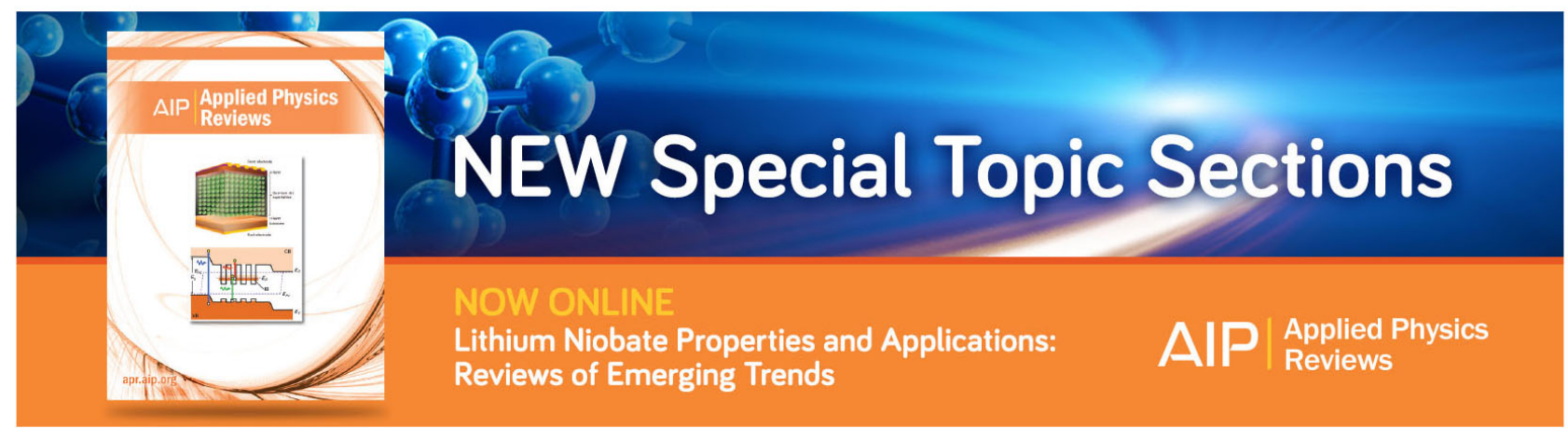




\title{
A corpuscular picture of electrons in chemical bond
}

\author{
Koji Ando \\ Department of Chemistry, Graduate School of Science, Kyoto University, Sakyo-ku, Kyoto 606-8502, Japan
}

(Received 5 February 2016; accepted 14 March 2016; published online 25 March 2016)

\begin{abstract}
We introduce a theory of chemical bond with a corpuscular picture of electrons. It employs a minimal set of localized electron wave packets with "floating and breathing" degrees of freedom and the spincoupling of non-orthogonal valence-bond theory. Its accuracy for describing potential energy curves of chemical bonds in ground and excited states of spin singlet and triplet is examined. ( 2016 AIP Publishing LLC. [http://dx.doi.org/10.1063/1.4944827]
\end{abstract}

\section{INTRODUCTION}

Modern concept of chemical bond has been prevailed by the molecular orbital (MO) model ${ }^{1,2}$ that is based on oneelectron orbitals with the mean-field approximation. Even the recently blooming density functional theory, ${ }^{3}$ whose genuine form should be independent of the MO concept, is practically based on the MO model in the Kohn-Sham scheme. The MO theory first solves a one-electron wave equation in the mean-field approximation and assigns electrons to the resultant MOs. The MOs reflect the molecular symmetry and are thus delocalized over the molecule or molecular moieties. They accommodate nodal structures of orbital phases that play important roles in determining chemical reactivity. ${ }^{4,5}$ Adequacy of the MO model has been endorsed by spectroscopies of photoelectron, ${ }^{6,7}$ electron scattering, ${ }^{8}$ and Penning ionization, ${ }^{9}$ some of which even attempt to observe MOs (more precisely Dyson orbitals).

While the MO picture emphasizes delocalized wave picture of one-electron orbitals, valence-bond (VB) model offers an alternative concept. ${ }^{10,11}$ The VB theory describes an electronic wave function as an antisymmetrized product of spatial and spin functions, the former consisting of a product of atomic orbitals (AOs). The "resonance structures" such as covalent and ionic ones provide intuitive understanding of chemical bond formation, reactivity, environmental effects, and so forth. In the full configuration-interaction (CI) limit with the same AO basis, the fully spin-coupled VB and the MO-CI are equivalent. In many cases, the VB model provides a compact and intuitive description of chemical phenomena.

Both MO and VB calculations have been based on linearcombination of atomic orbitals (LCAO) in which the AOs are clamped at the nuclear centers. By contrast, an alternative picture we present here is based on a "floating and breathing" wave packet (WP) model of corpuscular electrons. ${ }^{12,13}$ The model not only offers a distinct picture of static chemical bond but also has direct connection to the electron WP dynamics, an emerging arena with the recent advent of atto-second spectroscopic techniques. ${ }^{6,7,14-18}$

Related works on electronic ground states of $\mathrm{H}_{2}, \mathrm{LiH}$, $\mathrm{BeH}_{2}, \mathrm{CH}_{2}$, and $\mathrm{H}_{2} \mathrm{O}^{12,13}$ and on combined nuclear and electron WP molecular dynamics simulation of liquid $\mathrm{H}_{2}{ }^{19-21}$ have been reported. New results in this work are on high-spin (triplet) and excited states of $\mathrm{LiH}$. We also present results on the ground state of $\mathrm{NH}_{3}$, to supplement and compare with the previous work on the $\mathrm{AH}_{2}$ molecules.

\section{THEORY}

We employ an ordinary form of antisymmetrized product of spatial $(\Phi)$ and spin $(\Theta)$ functions for $N$-electron wave functions

$$
\Psi(1, \ldots, N)=\mathcal{A}\left[\Phi\left(\boldsymbol{q}_{1}, \ldots, \boldsymbol{q}_{N}\right) \Theta(1, \ldots, N)\right],
$$

in which $\mathcal{A}$ is the antisymmetriser and $\boldsymbol{q}_{i}$ represents the spatial coordinates of electrons. As usual, the spatial part assumes a product form of one-electron orbitals,

$$
\Phi\left(\boldsymbol{q}_{1}, \ldots, \boldsymbol{q}_{N}\right)=\phi_{1}\left(\boldsymbol{q}_{1}\right), \ldots, \phi_{N}\left(\boldsymbol{q}_{N}\right) .
$$

In the conventional VB method, the orbitals $\phi_{i}\left(\boldsymbol{q}_{i}\right)$ are constructed from the LCAO. By contrast, we employ "floating and breathing" minimal WPs of a form

$$
\begin{gathered}
\phi(\boldsymbol{q}, t)=\left(2 \pi \rho_{t}^{2}\right)^{-\frac{3}{4}} \exp \left[-\gamma_{t}\left|\boldsymbol{q}-\boldsymbol{r}_{t}\right|^{2}+i \boldsymbol{p}_{t} \cdot\left(\boldsymbol{q}-\boldsymbol{r}_{t}\right) / \hbar\right], \\
\gamma_{t}=\frac{1}{4 \rho_{t}^{2}}-\frac{i}{2 \hbar} \frac{\pi_{t}}{\rho_{t}},
\end{gathered}
$$

in which $\boldsymbol{r}_{t}$ and $\boldsymbol{p}_{t}$ are the WP center and its momentum, and $\rho_{t}$ and $\pi_{t}$ represent the WP width and its momentum. ${ }^{22}$ These dynamical variables are determined from the time-dependent or independent variational principle..$^{23,24}$

The spin part $\Theta(1, \ldots, N)$ consists of the spin eigenfunctions. ${ }^{2}$ We assume here a simple "perfect-pairing (PP)" form,

$$
\Theta=\theta(1,2), \ldots, \theta\left(N_{p}-1, N_{p}\right) \alpha\left(N_{p}+1\right), \ldots, \alpha(N),
$$

in which $\alpha$ and $\beta$ are the one-electron spin functions and $\theta(i, j)=(\alpha(i) \beta(j)-\beta(i) \alpha(j)) / \sqrt{2}$ is the singlet-pair function. The PP model has been chosen for simplicity and adequacy for the molecules studied here. We denote this model "WP-VBPP" or simply "WP-VB."

\section{CALCULATIONS AND DISCUSSION}

To calculate the potential energy surfaces in the ground state of a given total spin, the energy expectation $E$ $=\langle\Psi|H| \Psi\rangle /\langle\Psi \mid \Psi\rangle$ is minimized with respect to the center 


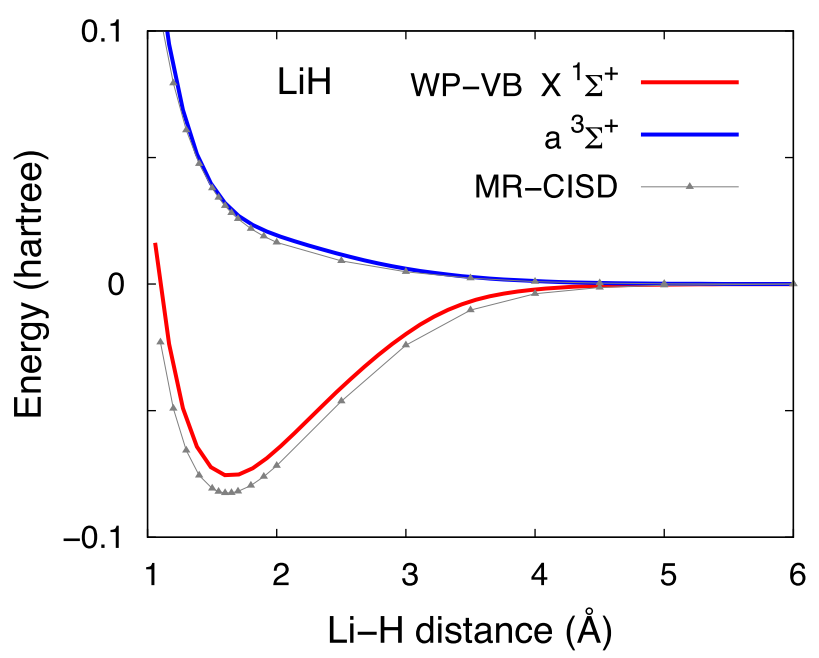

FIG. 1. Potential energy curves of singlet and triplet ground states, $X^{1} \Sigma^{+}$and $a^{3} \Sigma^{+}$, of LiH. WP-VB denotes the present floating and breathing minimal electron wave packet model with valence-bond spin-coupling. MR-CISD denotes multi-reference configuration-interaction with single and double excitations, with the cc-pVDZ basis set consisting of 27 atomic orbitals. The absolute energies from WP-VB and MR-CISD calculations were shifted by +7.109 hartree and +7.932 hartree, respectively, to match the dissociation limits.

and width variables $\boldsymbol{r}$ and $\rho$ of Eq. (3), with the momentum variables $\boldsymbol{p}$ and $\pi$ nullified. ${ }^{25}$ Figure 1 presents the resultant potential energy curves for the singlet $X^{1} \Sigma^{+}$and triplet $a^{3} \Sigma^{+}$ states of $\mathrm{LiH}$. They are compared to reference calculations of multi-reference CI with single and double excitations (MR-CISD) with the cc-pVDZ basis set that consisted of 27 primitive AOs in total. (We used the program GAMESS ${ }^{26}$ for the standard MO-based calculations.) By contrast, the WP-VB calculation employed the "minimal" basis, that is, only one WP per electron. The accurate energy curves were obtained by optimizing both the floating $(\boldsymbol{r})$ and breathing $(\rho)$ degrees of freedom, which accounted for the effects of polarization and so-called "dynamic" correlation, respectively, while the VB spin-coupling accounted for the "static" correlation. We attribute the WP breathing to the dynamic correlation as the WP width is affected by the direct interaction or collision with other WPs. This aspect is analogous to the effect of friction on the effective potential for a double-well potential coupled to harmonic bath. ${ }^{27}$

The minimal WP model offers a simple corpuscular picture of electrons in chemical bonds. Figures 2(a) and 2(b) display the electron WPs in the $X^{1} \Sigma^{+}$and $a^{3} \Sigma^{+}$states by circles with radius of the WP width $\rho$. They correspond to the electrons of, in the ascending order of the radius size, two $\mathrm{Li} 1 \mathrm{~s}, \mathrm{H} 1 \mathrm{~s}$, and Li 2s. While the former three WPs center around the nuclear positions, the "Li 2s" WP exhibits notable displacements. The pictures of the WPs correspond well with the conventional MOs in Figs. 2(c) and 2(d), two singly occupied alpha-spin MOs of the $a^{3} \Sigma^{+}$state, from the restricted open-shell Hartree-Fock calculation with the cc-pVDZ basis set. The HOMO and LUMO of the $X^{1} \Sigma^{+}$state look similar to them. These MOs are described by the linear combination of 27 AOs with various sizes and higher angular momenta for polarization. The corresponding electron distributions are (a)

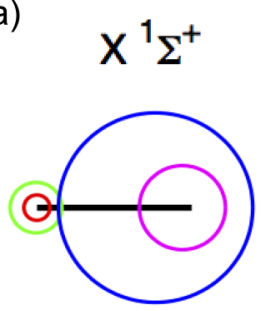

(c)

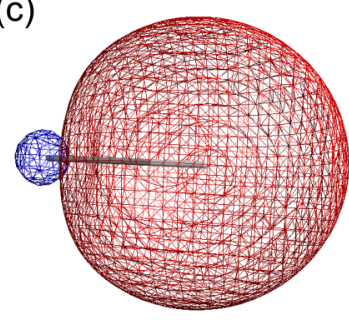

(b)

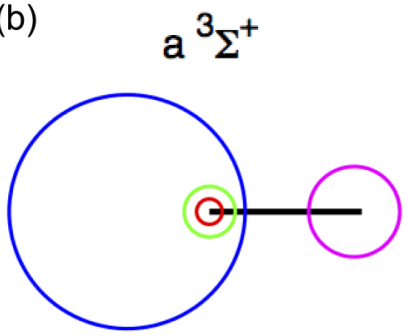

(d)

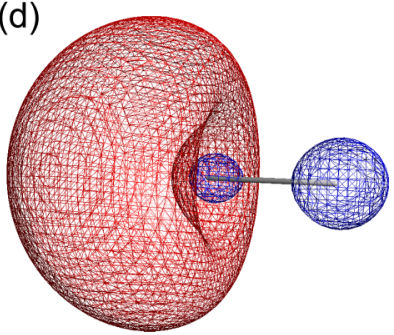

FIG. 2. Electron wave packets compared with molecular orbitals. (a) $X^{1} \Sigma^{+}$ state; (b) $a^{3} \Sigma^{+}$state. In (a) and (b), the radii of circles represent the width $(\rho)$ of electron wave packets that correspond to $\mathrm{Li} 1$ s (red and green), $\mathrm{H}$ 1s (magenta), and Li 2s (blue) electrons. (c) and (d) Two singly occupied alpha-spin molecular orbitals of $a^{3} \Sigma^{+}$state of $\mathrm{LiH}$ from restricted open-shell Hartree-Fock calculations with the cc-pVDZ basis set.

properly described by the floating and breathing minimal WPs in Figs. 2(a) and 2(b).

Next, we examine electronic excited states. In the MO framework, the most standard is the CI method. Another common approach is the equation-of-motion or Green function method that gives excitation energies from poles of electron propagator. $^{2}$ In the conventional VB framework, excited states are computed with non-orthogonal CI among independent spin-coupling configurations. ${ }^{10}$ Although this VB-CI method would be applicable with the present WP-VB scheme, we take an alternative route based on the idea of the propagator theory, since we have electron WP dynamics in perspective.

A complete method to construct the electron propagator in the present WP framework is via the coherent-state pathintegral formulation. ${ }^{23,28}$ It has been implemented in the initialvalue representation with the steepest-descent evaluation and Monte Carlo integration of the path-integral. ${ }^{24,29}$ However, here we take a simpler route: we construct potential energy surfaces for the electron WP motion and solve numerically the time-independent Schrödinger equation to obtain electronic excitation energies. This reduces to one or two dimensional calculations for the case of $\mathrm{LiH}$ as follows. From a preliminary normal-mode analysis, we found that the motion of a particular electron WP, which corresponds to the $\mathrm{Li} 2 \mathrm{~s}$ electron, dominates the lowest energy excitations. We thus shifted the center of that WP along the molecular axis to construct a potential for that electron motion. Numerical solution of the Schrödinger equation in this potential gives the excitation energies of the $\Sigma$ states. Similarly, the WP center was shifted perpendicular to the molecular axis for the $\Pi$ states.

Figure 3(a) presents the resultant potential energy curves for the excited $A^{1} \Sigma^{+}, B^{1} \Pi, b^{3} \Pi$, and $c^{3} \Sigma^{+}$states of $\mathrm{LiH}$. Despite the simplicity of calculation, the potential curves in 

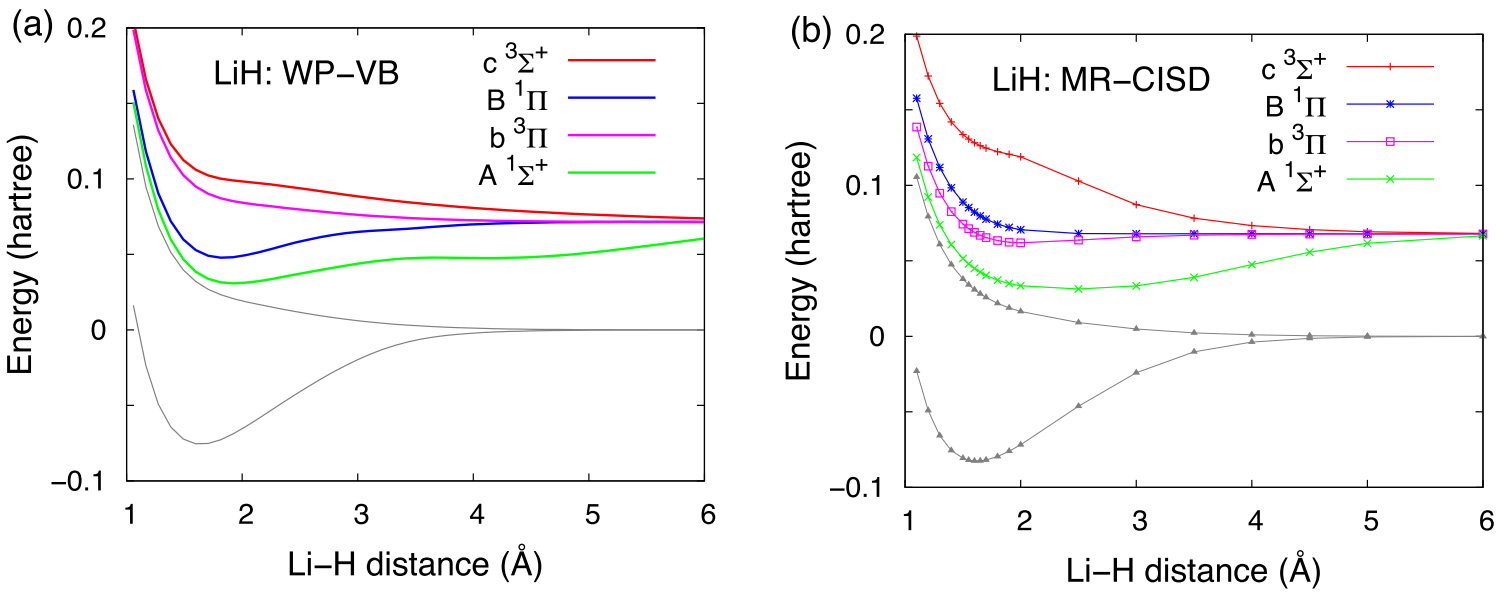

FIG. 3. Potential energy curves of $A^{1} \Sigma^{+}, B^{1} \Pi, b^{3} \Pi, c^{1} \Sigma^{+}$excited states of LiH. (a) The present electron wave packet calculation. (b) Multi-reference configuration-interaction with single and double excitations with the cc-pVDZ basis set. The shifts of absolute energies are identical to those indicated in the caption of Fig. 1.

Fig. 3(a) exhibit semi-quantitative accuracy in comparison with the MR-CISD reference displayed in Fig. 3(b). For the $A^{1} \Sigma^{+}$state, we have double-checked the results by constructing a two-dimensional potential energy surface for the motions of two electron WPs corresponding to the $\mathrm{H} 1 \mathrm{~s}$ and the Li $2 \mathrm{~s}$, because their overlap is apparent in Fig. 2(a) for the $X^{1} \Sigma^{+}$state. However, this additional calculation did not affect notably the results in Fig. 3(a). We have confirmed the reason that these degrees of freedom are well separated in terms of both the shape and curvature of the potential surface, as the $\mathrm{H} 1 \mathrm{~s}$ WP is much more tightly bound around the nucleus than the Li 2s WP. Although the WP-VB excited state curves in Fig. 3 demonstrate semi-quantitative accuracy, the incorrect energy ordering of the $\Pi$ states is noted. We anticipate that this will be remedied by more elaborate calculation of the electron propagator with the coherent-state path-integral formulation.

Finally, we present calculations on the ground state of a polyatomic molecule, $\mathrm{NH}_{3}$, whose conventional description
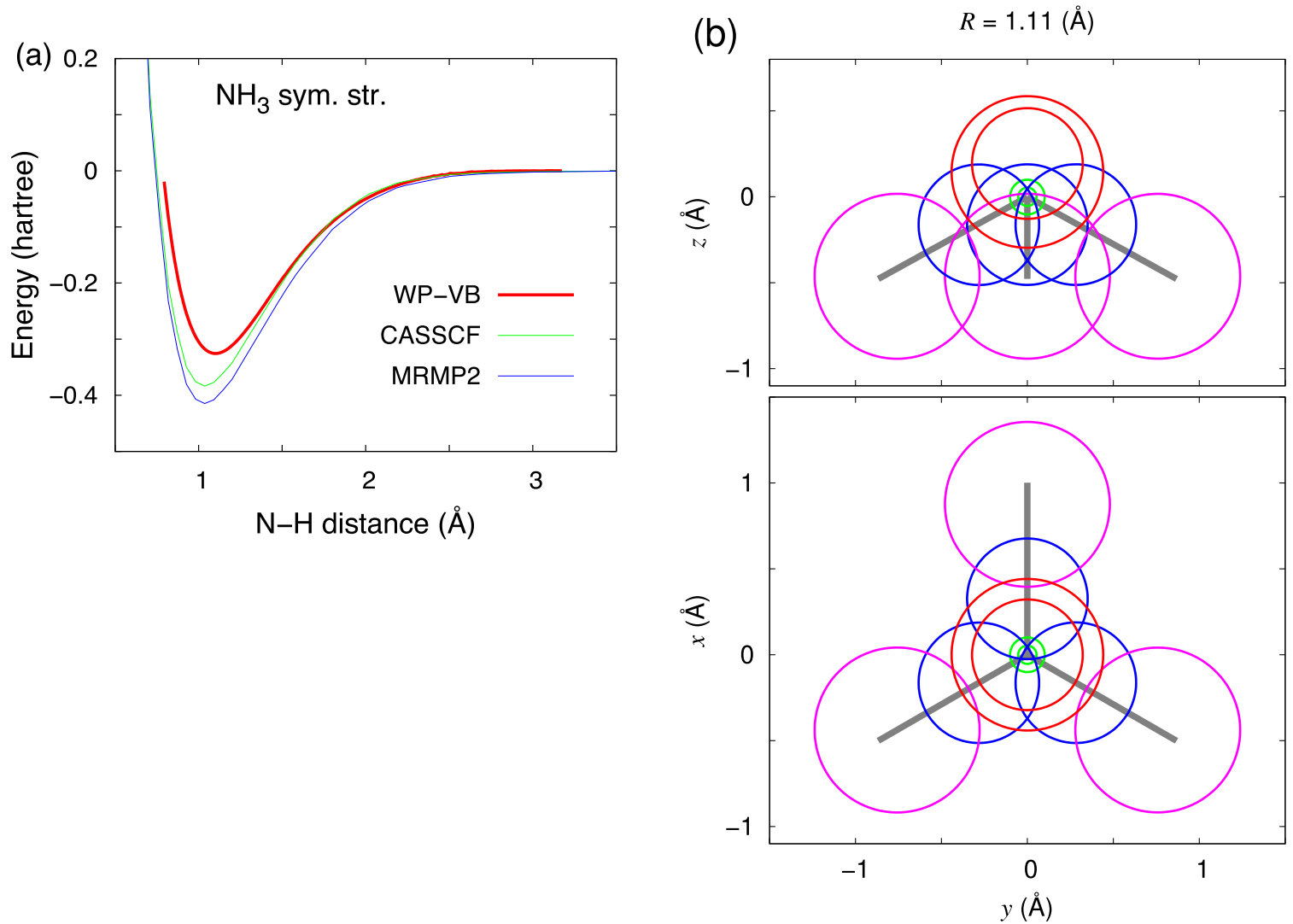

FIG. 4. Potential energy curves and electron wave packets for $\mathrm{NH}_{3}$. (a) Ground state potential energy curves along the N-H symmetric stretch coordinate of $\mathrm{NH}_{3}$, comparing the present electron wave packet valence-bond (WP-VB) model, complete active space self-consistent field (CASSCF), and multi-reference second-order Møller-Plesset perturbation theory (MRMP2), the latter two with the cc-pVDZ basis set. (b) Electron wave packets for $\mathrm{NH}_{3}$ with the radius of circles representing the wave packet widths $(\rho)$. 
involves nitrogen $2 \mathrm{p}$ AOs for the $\mathrm{NH}$ bonds and lone-pair electrons. Corresponding results for the $\mathrm{AH}_{2}$-type molecules, $\mathrm{BeH}_{2}, \mathrm{CH}_{2}$, and $\mathrm{H}_{2} \mathrm{O}$, have been reported previously. ${ }^{13}$ Here, we supplement the argument with the representative $\mathrm{AH}_{3}$ molecule. Figure 4(a) demonstrates that the potential energy curve of $\mathrm{NH}_{3}$ is properly described although the bond length is rather overestimated that would be related to the underestimate of the binding energy. Figure 4(b) displays how the electrons in NH bonds and lone-pair are described by the spherical WPs. The key is, as noted for Fig. 2, that the floating WPs account for the polarization of electronic wave function, for which the conventional LCAO scheme requires AOs of higher angular momenta as the AOs are clamped at the nuclear centers. Their nodal structures are not essential for generally nodeless total electronic wave functions in the ground state.

\section{CONCLUDING REMARKS}

Now we consider that the major bottleneck for applications to polyatomic molecules is not fundamental but mostly technical. In order to improve quantitative accuracy, the first task will be to employ more flexible WPs than the spherical ones. This will complicate the evaluation of two-electron integrals, but we have already applied ellipsoidal WPs to nuclear WP simulation of liquid water. ${ }^{30}$ Another task would be extension to multi-configuration spin-couplings that will be needed for some chemical reactions. ${ }^{10}$ Nonetheless, only a few spin-coupling patterns will suffice in practice for essential picture, especially due to the flexibility of floating and breathing WPs. Works on these are now under way and will be reported in due course.

\section{ACKNOWLEDGMENTS}

This work was supported by KAKENHI Nos. 26248009 and 26620007.
${ }^{1}$ A. Szabo and N. S. Ostlund, Modern Quantum Chemistry (Dover, New York, 1996).

${ }^{2} \mathrm{R}$. McWeeny, Methods of Molecular Quantum Mechanics (Academic, London, 1992).

${ }^{3}$ R. G. Parr and W. Yang, Density Functional Theory of Atoms and Molecules (Oxford University, Oxford, 1989).

${ }^{4}$ K. Fukui, Acc. Chem. Res. 4, 57 (1971).

${ }^{5}$ R. B. Woodward and R. Hoffmann, Angew. Chem., Int. Ed. Engl. 11, 781 (1969).

${ }^{6}$ J. Itatani, J. Levesque, D. Zeldler, H. Niikura, H. Pépin, J. C. Kleffer, P. B. Corkum, and D. M. Villeneuve, Nature 432, 867 (2004).

${ }^{7}$ O. Smirnova, Y. Mairesse, S. Patchkovskii, N. Dudovich, D. Villeneuve, P. Corkum, and M. Y. Ivanov, Nature 460, 972 (2009).

${ }^{8}$ M. Yamazaki, K. Oishi, H. Nakazawa, C. Zhu, and M. Takahashi, Phys. Rev. Lett. 114, 103005 (2015).

${ }^{9}$ K. Ohno, Bull. Chem. Soc. Jpn. 77, 887 (2004).

${ }^{10}$ S. S. Shaik and P. C. Hiberty, A Chemist's Guide to Valence Bond Theory (Wiley, New Jersey, 2008).

${ }^{11} \mathrm{~L}$. Pauling, The Nature of the Chemical Bond (Cornell University Press, New York, 1960).

${ }^{12}$ K. Ando, Bull. Chem. Soc. Jpn. 82, 975 (2009).

${ }^{13}$ K. Ando, Chem. Phys. Lett. 523, 134 (2012).

${ }^{14}$ M. F. Kling and M. J. J. Vrakking, Annu. Rev. Phys. Chem. 59, 463 (2008).

${ }^{15}$ F. Krausz and M. Ivanov, Rev. Mod. Phys. 81, 163 (2009).

${ }^{16}$ T. Kato, T. Oyamada, H. Kono, and S. Koseki, Prog. Theor. Phys. Suppl. 196, 16 (2012).

${ }^{17}$ M. Vacher, D. Mendive-Tapia, M. J. Bearpark, and M. A. Robb, Theor. Chem. Acc. 133, 1505 (2014).

${ }^{18}$ M. J. T. Oliveira, B. Mignolet, T. Kus, T. A. Papadopoulos, F. Remacle, and M. J. Verstraete, J. Chem. Theory Comput. 11, 2221 (2015).

${ }^{19}$ K. Hyeon-Deuk and K. Ando, J. Chem. Phys. 140, 171101 (2014).

${ }^{20}$ K. Hyeon-Deuk and K. Ando, Phys. Rev. B 90, 165132 (2014).

${ }^{21}$ K. Hyeon-Deuk and K. Ando, J. Chem. Phys. 144, 171102 (2015).

${ }^{22}$ F. Arickx, J. Broeckhove, E. Kesteloot, L. Lathouwers, and P. van Leuven, Chem. Phys. Lett. 128, 310 (1986).

${ }^{23}$ H. Kuratsuji, Prog. Theor. Phys. 65, 224 (1981).

${ }^{24}$ K. Ando, Chem. Phys. Lett. 591, 179 (2014).

${ }^{25}$ Y. Tsue, Prog. Theor. Phys. 88, 911 (1992).

${ }^{26}$ M. W. Schmidt, K. K. Baldridge, J. A. Boatz, S. T. Elbert, M. S. Gordon, J. H. Jensen, S. Koseki, N. Matsunaga, K. A. Nguyen, S. J. Su et al., J. Comput. Chem. 14, 1347 (1993).

${ }^{27}$ K. Ando, J. Chem. Phys. 121, 7136 (2004).

${ }^{28}$ Coherent States: Applications in Physics and Mathematical Physics, edited by J. R. Klauder and B. S. Skagerstam (World Scientific, Singapore, 1985).

${ }^{29}$ K. G. Kay, Annu. Rev. Phys. Chem. 56, 255 (2005).

${ }^{30}$ J. Ono and K. Ando, J. Chem. Phys. 137, 174503 (2012). 